\title{
Supervised Learning Based Stereo Matching Using Neural Tree
}

\author{
Sanjeev Kumar ${ }^{1}$, Asha Rani ${ }^{2}$, Christian Micheloni ${ }^{2}$, and Gian Luca Foresti ${ }^{2}$ \\ 1 Department of Mathematics, IIT Roorkee, Roorkee 247667, India \\ 2 Department of Mathematics and Computer Science, University of Udine, \\ Udine 33100, Italy
}

\begin{abstract}
In this paper, a supervised learning based approach is presented to classify tentative matches as inliers or outliers obtained from a pair of stereo images. A balanced neural tree (BNT) is adopted to perform the classification task. A set of tentative matches is obtained using speedup robust feature (SURF) matching and then feature vectors are extracted for all matches to classify them either as inliers or outliers. The BNT is trained using a set of tentative matches having ground-truth information, and then it is used for classifying other sets of tentative matches obtained from the different pairs of images. Several experiments have been performed to evaluate the performance of the proposed method.
\end{abstract}

Keywords: Neural Tree, Stereo Vision, Tentative Matches, Supervised Learning.

\section{Introduction}

One of the major challenges in wide baseline stereo images is the detection and removal of the outliers from a set of tentative matches due to its applicability in many vision applications such as construction of mosaic images, rectification of stereo images, change detection etc. Most of these applications require the estimation of a transformation matrix called homography. Such a homogarphy is estimated by minimizing a linear or nonlinear function for a given number of matching points. In this context, the nature of the homography estimation process is very sensitive with respect to false matches. Most of the image matching algorithms [1] contain three phases. In the first phase features are detected in both images in such a way that the detected features have similar appearance in different images (i.e., invariant [2]). Then, feature descriptors are computed as the signatures of the features. In the third phase the features of the first image are compared to the features of the second image. The comparison is performed using a suitable distance measure on the descriptors, and the tentative matches are ordered by similarity.

Scale invariant feature transform called as 'SIFT' [2] and speedup robust features (SURF) 3] based descriptors are very popular and effective approaches for obtaining the pairs of matching points between stereo images. However, when a 
wide baseline stereo system is used, the number of outliers becomes considerable. Therefore an extra phase is required to classify and remove such outliers from tentative matches. In classical approaches, these outliers are removed by fitting an affine or perspective transformation model to the top tentative matches. The model is then used to classify each tentative match as an inlier or outlier. All RANdom SAmple Consensus (RANSAC) based approaches 45] come under this category. Recently, an affine invariant model based approach has been proposed to classify tentative matches as inliers or outliers [6] with an advantage that it does not rely on the typical model generation as in RANSAC-based methods. These approaches are unsupervised in nature and not very effective when a large number of outliers are present in the respective set of tentative matches as in the case of wide baseline stereo images shown in Fig. 1 In such a scenario, the use of supervised learning based approaches is a more sensible choice in place of unsupervised techniques.
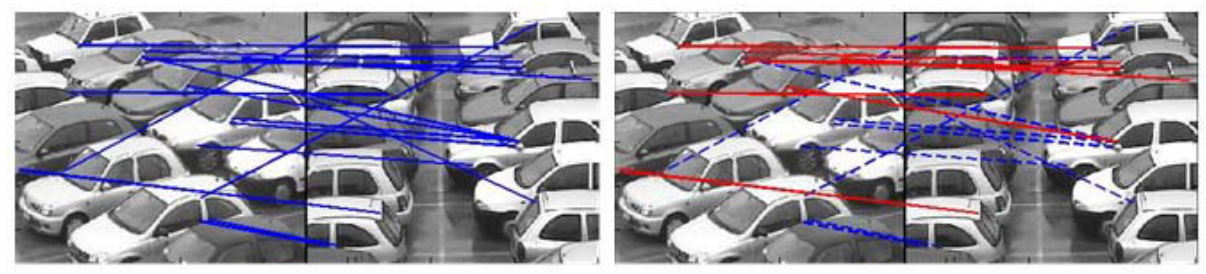

Fig. 1. Wide baseline stereo image matching. A set of tentative matches using SURF (left), Outlier detection using RANSAC (right), where blue dotted line represents detected outliers from the set of tentative matches.

In order to establish the point matching between a pair of stereo images, few supervised learning based methods have been proposed [7/8], where different machine learning tools like support vector machines (SVM) and artificial neural networks (ANN) have been used. These approaches are effective for performing the matching on the edges in stereo images, but at the same time have few drawbacks like the selection of an optimal architecture of the neural network for getting faster convergence with good accuracy. Moreover, these approaches are based on the matching of various edges between two images which makes them computationally expensive.

In this work, we are proposing a modified version of a neural tree 9 called balanced neural tree (BNT) for classifying inliers and outliers from a set of tentative matches (see Fig. 22). This BNT is composed by simple (single layer) perceptrons at its various nodes. Instead of local matching between images such as edge matching [8, we use a set of tentative matches obtained with SURF descriptors. For each tentative match in a set, a five dimensional feature vector (or pattern) is extracted and such a pattern is used as the input of the BNT. The $\mathrm{BNT}$ is trained in such a way that it achieves a balanced structure for better classification in less time. The proposed method has some advantages over existing 


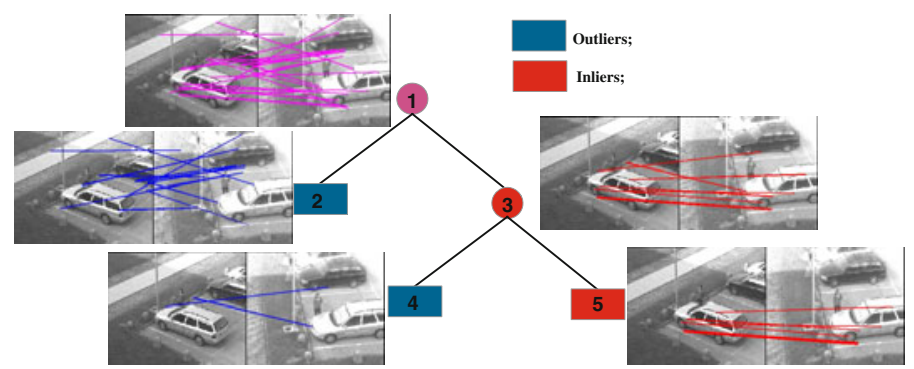

Fig. 2. An overview of the proposed framework: classification of tentative matches as inliers or outliers with neural tree's growth

unsupervised as well as supervised learning based approaches like its classification accuracy does not depend on the nature of data sets as in RANSAC and no need to decide an optimal architecture of network as in mutilayer perceptrons based approaches.

\section{Features and Attributes Extraction}

A pattern is generated corresponding to each tentative match based on the similarity between the descriptors and their respective local neighborhood in two images. Two different steps are required to obtain the necessary information for classifying a tentative match as inlier or outlier: (1) extraction of tentative matches from the pairs of stereo images, and (2) construction of feature vectors (or patterns) from these tentative matches.

The tentative matches are extracted using the SURF descriptor [3] due to its almost real time performance. The SURF detector is based on the Hessian matrix [10, but uses a very basic approximation like Laplacian-based detector. It relies on integral images to reduce the computation time and therefore called the 'FastHessian' detector. The descriptor, on the other hand, describes a distribution of Haar-wavelet responses within the neighborhood of points of interest. Again, integral images are exploited for speed. Moreover, in SURF descriptors, only 64 dimensions are used for reducing the time of feature computation and matching, as well as increasing the robustness. The first step consists of fixing a reproducible orientation based on information from a circular region around the point of interest. Then, we construct a square region aligned to the selected orientation, and extract the SURF descriptor from it.

For each pair of descriptors belonging to a set of tentative matches, a total five different measures (the magnitude of the gradient vector, the direction of the gradient vector, the Laplacian, the variance and the mean) are computed. In this process, the gray levels of a central pixel (descriptor) and its eight immediate neighbors are used with the help of a $3 \times 3$ window. To find the gradient magnitude of the central pixel, we compare the gray level differences from the four pairs of opposite pixels in the 8-neighborhood, and the largest difference is taken as the gradient magnitude. The gradient direction of the central pixel is the direction out 
of the eight principal directions whose opposite pixels yield the largest gray level difference and also points in the direction which the pixel gray level is increasing. It is measured in degrees and a quantification followed by a normalization is performed as in [8]. The Laplacian is computed by using the corresponding Laplacian operator over the eight neighbors of the central pixel. The variance indicates the dispersion of the nine gray level values in the eight-neighborhood computed after a normalization process based on a linear regression.

Using the above described procedure, two different five dimensional vectors $x_{l}$ and $x_{r}$ are obtained corresponding to a given tentative match. Here, the components are the measures and the sub-indices $l$ and $r$ indicates that either vectors are corresponding to the left or right images, respectively. By taking the absolute difference of these two vectors $x=\left|x_{l}-x_{r}\right|$, we obtain the required feature vector or pattern $x$, whose components are corresponding to the differences for the module of the gradient vector, the direction of the gradient vector, the Laplacian, the variance and the mean, respectively.

\section{Proposed Classifier: Balanced Neural Tree}

Neural trees (NTs) have been developed to overcome the limitations of decision trees and neural networks based classification approaches. A neural tree grows during the learning phase and so it does not require any priori information about the network architecture (like number of hidden neurons or hidden layers). Moreover, it does not require any exhaustive search as used in training algorithms for decision trees. The first neural tree has been proposed in [11], whose internal nodes are represented by attribute tests and leaf nodes by perceptrons. Later, in [12, attribute tests at internal nodes are replaced by perceptrons. In some cases, a single layer perceptron can stuck in local extremals resulting in poor generalisation of the patterns which leads to a unbalanced or nonconvergent tree building process. In [9], a solution has been provided to this problem by introducing split nodes. Although split nodes assure the convergence of tree building process, but can generate a deep and unbalanced tree. Here, we propose a new tree architecture, called balanced neural tree (BNT), able to reduce the size of the tree with good classification accuracy. Two main novelties are proposed to achieve such a result: 1) balance the structure of the tree by substituting the current trained perceptron with a new perceptron if the current trained perceptron largely misclassifies the current training set into reduced number of classes, and 2) adopt a new criterion for the removal of tough training patterns that generate an over-fitting problem.

Learning algorithm of the proposed BNT is inherited from [9]. However, some novelties are introduced in order to overcome the above mentioned drawbacks. Let $S=\left\{\left(x_{j}, c_{i}\right) \mid j=1, \ldots, n \wedge i \in[1, C]\right\}$ be the training set containing $n$ number of $k$-dimensional $x_{j}$ patterns belonging to a class $c_{i}$ out of the possible $C$ classes. The learning phase of the BNT is described in the Algorithm 1 Let $S^{\prime}$ be the local training set at the current node $v, Q_{v}$ and $Q_{S}$ be the queues holding the nodes and corresponding local training sets to be learned. 


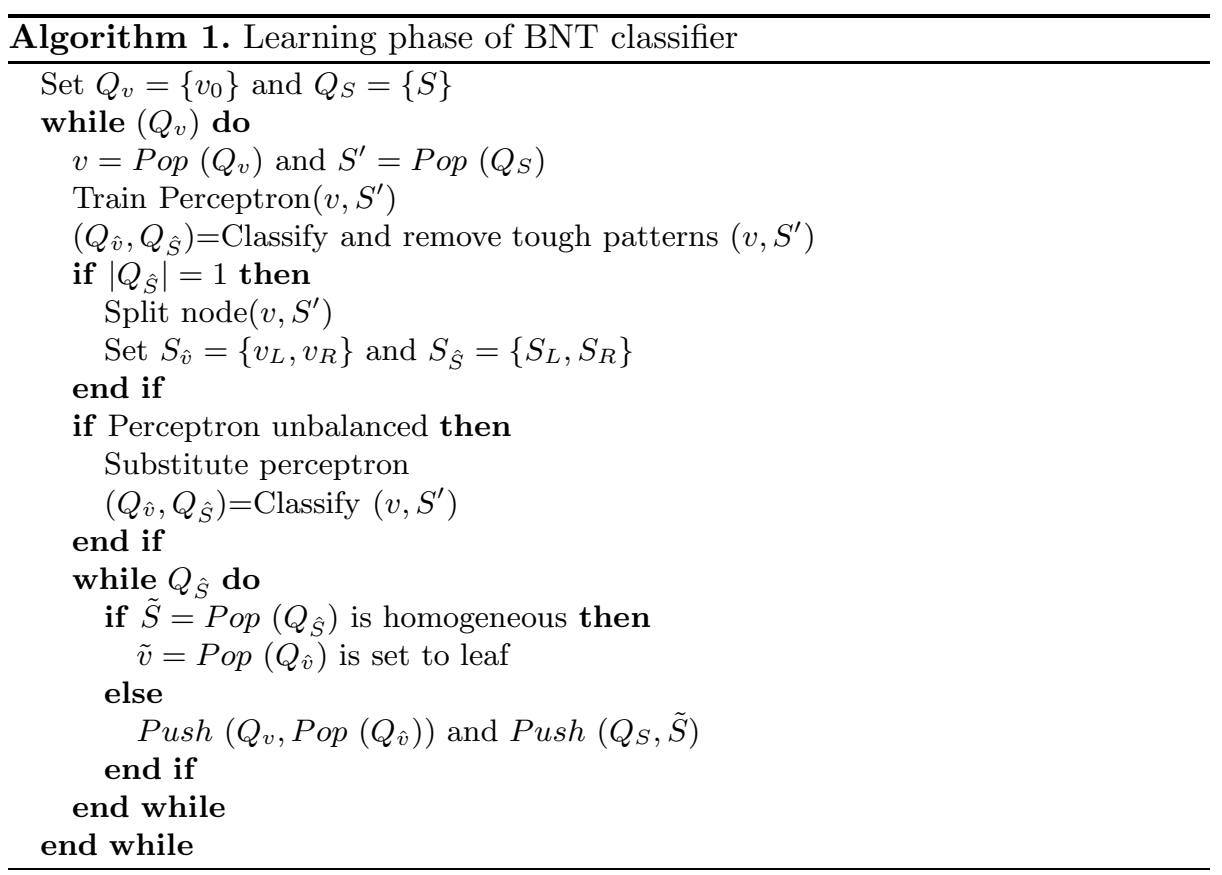

In the Algorithm 11 $v_{0}$ represents the root node, $S$ is the training set at root node, $Q_{\hat{v}}$ and $Q_{\hat{S}}$ are the local queues to hold $\hat{v}$ and $\hat{S}$, respectively. The descriptions of the functions "Train perceptron", "Classify and remove tough patterns", "Split", and "Substitute Perceptron" mentioned in the above learning algorithm are explained below:

Train Perceptron $\left(v, S^{\prime}\right)$. It trains a single layer perceptron at node $v$ on the training set $S^{\prime}$. The perceptron learns until the error is not reducing any more for a given number of iterations. A trained perceptron generates $\left(o_{1}, \ldots o_{c}\right)$ activation values.

Classify and remove tough patterns $\left(v, S^{\prime}\right)$. It assigns the pattern to the class corresponding to the highest activation value and removes the patterns that are tough to classify. In other words, it divides $S^{\prime}$ in to $\hat{S}=$ $\left\{S_{1}, \ldots, S_{c}\right\}, \quad c \leq C$ subsets and generates next level of child nodes $\hat{v}=$ $\left\{v_{1}, \ldots v_{c}\right\}, \quad c \leq \bar{C}$ corresponding to $\hat{S}$. Returns $(\hat{v}, \hat{S})$.

$\operatorname{Split}\left(v, S^{\prime}\right)$. It divides the current training set into two parts $S_{L}$ and $S_{R}$ and generates corresponding child nodes $v_{L}$ and $v_{R}$.

Substitute Perceptron. It substitutes an unbalanced perceptron with a new perceptron whose weights are initialized in such a way that the hyperplane generated passes through the barycentre of the training set.

The studied strategy bases its decision about which splitting criteria must be adopted on the basis of the classification errors. In particular, at each node the global classification error is computed as $E_{t}=1-\left(K_{c} / K_{t}\right)$, where $K_{c}$ is the number of correctly classified patterns and $K_{t}$ is the total number of patterns 
presented at the current node. In addition, to better localise the error among the classes, for each class a misclassification error is computed as $E_{i}=1-\left(K_{c_{i}} / K_{t_{i}}\right)$, where $K_{c_{i}}$ is the number of correctly classified patterns of class $i$, and $K_{t_{i}}$ is the total number of patterns classified as class $i$. During training, the criterion to judge whether a perceptron classification is acceptable is given as:

$$
E_{t}>\frac{E_{0}}{m} \quad \text { and } \quad\left(E_{\max }-E_{\min }\right)>E_{t}
$$

where $m$ is the number of classes at the current node, $E_{\text {max }}=\max _{i}\left\{E_{i}\right\}$, $E_{\text {min }}=\min _{i}\left\{E_{i}\right\}$ and $E_{0}$ represents the initial error. If a perceptron is not able to separate the training set according to above mentioned criterion, then such a classification is not accepted and the corresponding perceptron is replaced with a specially designed perceptron that distribute the training set among all the classes equally. This kind of perceptron is generated by passing the splitting hyperplane through the barycenter of training set. The third kind of splitting is done when the perceptron fails to separate the patterns. For such a purpose a splitting rule 9] is considered to divide the data in two groups with almost equal cardinality. Concerning the second novelty of the proposed training algorithm, it has been noticed that even a well trained perceptron is not able to classify certain patterns. Such patterns are responsible for over-fitting in the training set. Such patterns are removed from the training set based on two facts, i.e., the probability of a pattern belonging to a class and the total classification error of the perceptron.

The classification probability is modelled by normalizing the activation values so that they form a distribution. The uncertainty between the two classes is given as $h_{i j}=\left|P\left(c_{i} \mid \mathbf{x}\right)-P\left(c_{j} \mid \mathbf{x}\right)\right|$, where $P\left(c_{i} \mid \mathbf{x}\right)$ and $P\left(c_{j} \mid \mathbf{x}\right)$ are the probabilities of pattern $\mathbf{x}$, belonging to classes $c_{i}$ and $c_{j}$ respectively. Let $P\left(c_{\max 1} \mid \mathbf{x}\right)$ and $P\left(c_{\max 2} \mid \mathbf{x}\right)$ be the maximum and second maximum classification probabilities, thus representing the two most probable classes to which $\mathbf{x}$ should belong, then $h_{\max }=\left|P\left(c_{\max 1} \mid \mathbf{x}\right)-P\left(c_{\max 2} \mid \mathbf{x}\right)\right|$ is defined to represent the uncertainty of the trained perceptron.

Concerning the reliability of the perceptron classification, studying the behaviors of the nodes with respect to their depth, suggested to define the reliability $R=1 / m^{2}$. If the current total error $E_{t}$ is lower than the reliability factor $R$ then the perceptron can be considered reliable. The criterion to decide whether a pattern has to be removed from the training set or not, is based on the following rule:

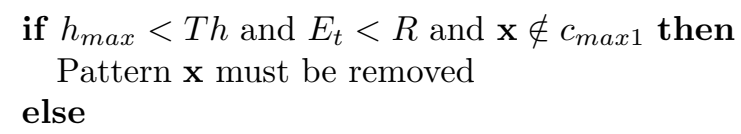

\section{Pattern $\mathbf{x}$ is included in $T S_{\max 1}$}

\section{end if}

Once the tree is build by learning the patterns, it can be used to classify the test patterns. The top to down traversal scheme [9] is used to classify the patterns. A pattern starts traversing the tree from the root node and traverse down until it reaches a leaf node. The next node to be considered on the path 
is decided by "winner-takes-all" rule. The class to be predicted is given by the leaf node reached by the pattern.

To perform the inlier and outlier classification on a set of tentative matches, BNT is trained on training data. In an online process, a set $S$ having $n$ pattern samples of tentative matches is classified into two classes inliers or outliers. The outputs of the system are two symbolic values $(+1,-1)$ each corresponding to one of the classes. During the classification process there are unambiguous and ambiguous matches, depending on whether a given left image segment corresponds to one and only one, or several right image segments, respectively. In any case, the decision about the correct match is made by choosing the pair with the higher activation value.

\section{Experimental Results}

Our aim is to check the performance of the proposed inliers-outliers classification method on different pairs of images. To do this, we have captured thirty pairs of stereo images of six different scenes. These image pairs are captured with a calibrated and wide baseline stereo cameras setup under varying illumination and baseline distance. Tentative matches are extracted from all image pairs using SURF descriptor followed by the earlier described process for obtaining respective feature vectors. All these feature vectors are put into six different sets (TM1TM6) of tentative matches based on each scene. To check the performance of the proposed method, we have compared our classification results with different unsupervised (RANSAC [4] and affine invariant 6]) and supervised (multilayer perceptron [13] and AdaBoostM1 [14]) learning based methods. Here, employed multilayer perceptron has been composed by one hidden layer having four nodes and trained with a backpropagation learning algorithm.

Since we know the calibration parameters of the employed cameras, the groundtruth information have been extracted for the tentative matches (i.e., whether a match belong to inlier or outlier). The objective behind having the ground-truth is to know the percentage of correctly classified matches from tentative matches, i.e., evaluation of the accuracy of the proposed algorithm.

First, the set TM1 having total 457 tentative matches is used as a training set. In this set, classes (inlier or outlier) are assigned to each match and then this data is used for tree learning/building procedure. Once the tree is built, it is used to classify the tentative matches from other five sets (TM2-TM5) obtained with the images of different scene as well as illumination. This procedure has

Table 1. Classification accuracy using simple training strategy

\begin{tabular}{|c|c|c|c|c|}
\hline Data Sets & Proposed BNT & Multilayer perceptron & AdaBoostM1 14 & RANSAC \\
\hline TMI & 98.96 & 99.20 & 99.05 & 94.40 \\
\hline TM2 & & 98.50 & 97. & 91.1 \\
\hline TM3 & & 97. & 96.95 & 94.54 \\
\hline TM4 & 97.42 & 97.79 & 96.64 & 95.21 \\
\hline TM5 & & 97.41 & & 93.70 \\
\hline TM6 & 97.94 & 98.19 & 97.82 & 91.40 \\
\hline
\end{tabular}


been repeated according to leave-one-out strategy, i.e. training on a data set and testing on the rest data sets. Finally, average of the classification accuracies of all data set obtained in five runs are taken. For a detailed quantitative analysis, these results showing the classification accuracy of the patterns belonging to these sets using above mentioned methods are given in Table 1 in terms of the percentage of correctly classified patterns.

From this table, it is clear that the proposed method perform better than the RANSAC for each data set (TM2-TM6). It is also worth to notice that it performed better than multilayer perceptron (MLP) in case of data sets TM2, TM3 and TM5, while for data sets TM1, TM4 and TM6, the performance of the MLP is better than our proposed method. However, the main advantage of the proposed method over MLP is that there is no need to decide the network architecture for getting optimal performance. It is also noticeable that the proposed method performed better than the AdaBoost classifier except the data set TM1, where the performance are quite close and comparable. For a visual representation of these results, Fig. 3 represents the two stereo-image pairs along with classification results obtained using the proposed and RANSAC algorithms.
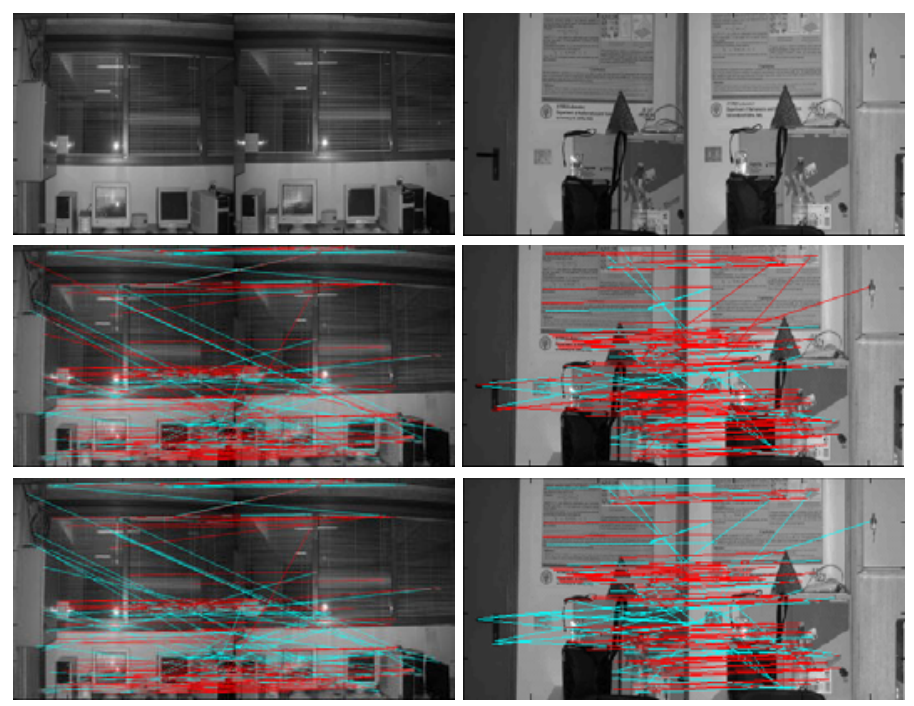

Fig. 3. Classification of tentative matches as inliers (red lines) and outliers (cyan lines). First row: two different stereo image pairs; second row: classification using RANSAC, and third row: classification using proposed BNT classifier.

Moreover, we have adopted an iterative process for the training of neural tree. First, the neural tree has been trained using data set TM1 and employed to obtain $\mathrm{O}(\mathrm{TM} 2)$ (where 'O' represents output) by classifying TM2. The classification results of TM2 has been combined with TM1, and again the neural tree has been trained using the combined (TM1+O(TM2)) training set. This 
Table 2. Classification accuracy using iterative training strategy

\begin{tabular}{|c|c|c|c|c|}
\hline Sets & Training Patterns & Testing Patterns & Proposed BNT & Multilayer perceptron \\
\hline TM1 & 457 & 457 & 100.0 & 100.0 \\
TM2 & 457 & 300 & 98.00 & 98.34 \\
TM3 & 757 & 495 & 98.94 & 98.90 \\
TM4 & 1252 & 520 & 99.60 & 99.65 \\
TM5 & 1772 & 540 & 99.38 & 99.42 \\
TM6 & 2312 & 614 & 99.38 & 99.50 \\
\hline
\end{tabular}
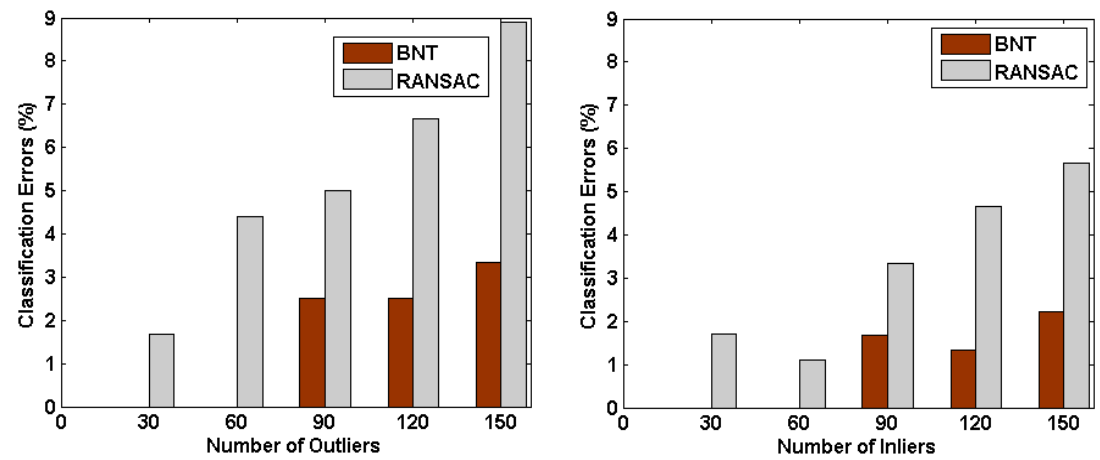

Fig. 4. Classification error in presence of different number of outliers/inliers in tentative matches

procedure has been repeated up to classification of TM6. The classification results obtained by adopting this iterative training strategy are given in Table 2 , It is worth to notice that the result obtained with iterative training strategy is better than the earlier one in case of supervised learning algorithms. The reason behind this improvement is the better adoption of different factors like changes in the illumination and the scene for the training data of the neural tree. For observing the effect of the increasing numbers of outliers/inliers in the classification of tentative matches, we have conducted an experiment by considering different number of inliers or outliers in a set of tentative matches. In Fig. 4, a graph is shown between the classification error and increasing number of outliers, while keeping the fixed number of inliers. Initially, we considered 30 inliers (fixed) and 0 outliers in our set of tentative matches taken from data set TM6. This set has been classified by the BNT (trained on TM1) and RANSAC. Then, we have repeated this process five times by adding 30 outliers each time in the set of tentative matches and obtained the results. This experiments has been performed on five different samples and average of the classification errors are calculated. It has been noticed that the classification error increases very slowly in case of BNT, while is increases gradually in the case of RANSAC. A similar kind of results (see Fig. 4) by increasing number of inliers in the set of tentative matches while fixing the number of outliers as constant. Again, the variation in the error has been found very small in the case of BNT while it is more in the case of RANSAC. 
It is also observed that most of the times only inliers are misclassified in the case of BNT classifier. It means that the inliers detected by the proposed method can be used for many sensitive operations in stereo vision like homography estimation, image rectification etc, as there is very minor chances for having an incorrect match. Here, it is also worth to mention that the proposed method gives a real time performance in terms of computational time.

\section{Conclusions}

We have proposed a new method to classify feature correspondences as inliers or outliers in stereo images. The proposed approach does not rely on the typical model generation and test approach used in RANSAC-based methods or in other unsupervised learning based classification approaches. A simple perceptron based neural tree has been employed to classify the tentative matches as inliers or outliers. The advantage of using neural tree over multilayer perceptron or other network architecture is that there is no need to decide an optimal network structure such as number of hidden layers or number of nodes in each hidden layer. It has been found that the proposed approach gives a very good result in different cases such as in different illuminations as well as scenes.

\section{References}

1. Mikolajczyk, K., Schmid, C.: A performance evaluation of local descriptors. IEEE Trans. Pattern Anal. Mach. Intell. 27(10), 1615-1630 (2005)

2. Lowe, D.: Distinctive image features from scale-invariant keypoints. Int. Journal on Computer Vision 60, 91-110 (2004)

3. Baya, H., Essa, A., Tuytelaarsb, T., Gool, L.V.: Speed up robust features (surf). Computer Vision and Image Understanding 110(3), 346-359 (2008)

4. Torr, P., Zisserman, A.: Mlesac: a new robust estimator with application to estimating image geometry. Computer Vision and Image Understanding 78(1), 138-156 (2000)

5. Fischler, M.A., Bolles, R.: Random sample consensus: a paradigm for model fitting with applications to image analysis and automated cartography. Comm. ACM 24(6), 381-395 (1981)

6. Fleck, D., Duric, Z.: Affine invariant-based classification of inliers and outliers for image matching. In: Kamel, M., Campilho, A. (eds.) ICIAR 2009. LNCS, vol. 5627, pp. 268-277. Springer, Heidelberg (2009)

7. Pajares, G., Cruz, J.: Local stereovision matching through the adaline neural network. Pattern Recognition Letters 22, 1457-1473 (2001)

8. Pajares, G., Cruz, J.: Stereovision matching through support vector machines. Pattern Recognition Letters 24, 2575-2583 (2003)

9. Foresti, G., Pieroni, G.: Exploiting neural trees in range image understanding. Pattern Recognit. Lett. 19 (9), 869-878 (1996)

10. Mikolajczyk, K., Schmid, C.: Indexing based on scale invariant interest points. In: Int. Conf. on Computer Vision, vol. 1, pp. 525-531 (2001) 
11. Utgoff, P.E.: Perceptron tree: A case study in hybrid concept representation. Connection Science 1(4), 377-391 (1989)

12. Sankar, A., Mammone, R.: Neural Tree Networks. In: Neural Network: Theory and Application, pp. 281-302. Academic Press Professional, Inc., San Diego (1992)

13. Hall, M., Frank, E., Holmes, G., Pfahringer, B., Reutemann, P., Witten, I.: The weka data mining software: An update. SIGKDD Explorations 11(1) (2009)

14. Freund, Y., Schpire, R.: Experiments with a new boosting algorithm. In: Int. Conf. on Machine Learning, pp. 148-156. Morgan Kaufmann Pub. Inc., San Francisco (1996) 